\title{
Hausmittel bei Magen-Darm- Beschwerden: Vergessene Mittel aus der Hausapotheke und vom Wegesrand
}

In der Naturheilkunde hat die Verdauung eine grosse Bedeutung, denn der Mensch kann seine Nahrung nur verstoffwechseln, wenn seine Verdauungsleistung gut funktioniert. Aus diesem Grund werden in einer naturheilkundlichen Therapie Magen und Darm (mit-)behandelt, auch wenn andere Erkrankungen im Vordergrund stehen. In der Traditionellen Europäischen Naturheilkunde (TEN) werden die Verdauungs-, Resorptions- und Assimilationsvorgänge in der Kochungslehre (Coctio) beschrieben, die bildlich ausdrückt, dass der Mensch darauf angewiesen ist, dass er alles, was er an stofflicher und energetischer Nahrung einnimmt, verdauen ( «kochen») kann. Im Volksmund wird z.B. nach einem Streit oder einem schwierigen Gespräch gesagt «Das muss ich zuerst verdauen», was im übertragenen Sinn aufzeigt, dass der Mensch für sein physisches und psychisches Wohlbefinden darauf angewiesen ist, dass er eben «gut verdauen» kann $[1,2]$.

Auch in der Volksheilkunde hat die Behandlungvon Verdauungsbeschwerden eine lange Tradition. Dies aus drei Gründen:

(1) Weil Verdauungsbeschwerden häufig vorkommen,

(2) weil sie den Erkrankten sehr «plagen",

(3) weil die Beschwerden mit Naturheil- und Hausmitteln gut behandelt und gelindert werden können, sodass sich der betroffene Mensch bald wieder wohlfühlt.

Die Alltagsqualität ist bekanntlich direkt davon abhängig, wie die Ver- dauung arbeitet. Von Plinius ist überliefert [3]: «Es ist der Bauch, für dessen Befriedigung die meisten Menschen arbeiten. Es ist aber auch der Bauch, der für die meisten Menschen Leiden bringt.»

Eine kleine Auswahl an Heilpflanzen und Anwendungen bei MagenDarm-Beschwerden wird nachfolgend vorgestellt.

\section{Wärme und Ruhe}

Eine sehr einfache, vielleicht fast banale Form, Bauchweh und Blähungen zu lindern, ist eine Wärmeanwendung und das Einrichten einer Ruhezeit. Eine warme Bettflasche oder ein Kirschensteinsack auf dem Bauch hat schon manche Magen-Darm-Verstimmung in kurzer Zeit gelindert. Sich einen Moment hinlegen, wenn es im Bauch rumort und krampft, hilft erfahrungsgemäss sehr schnell. Dass Ruhe in unserer hektischen Zeit oft fehlt, ist bestimmt einer der Gründe, warum so viele Menschen an akuten und chronischen Magen-Darm-Beschwerden leiden.

\section{Heidelbeeren (Vaccinium myrtillus, Myrtilli fructus)}

Getrocknete Heidelbeeren sind ein altes, beinahe vergessenes Heilmittel, das auf milde Weise Durchfall zu behandeln vermag. Die Heidelbeere, die zu den Ericaceae (Heidekrautgewächse) zählt, wurde beispielsweise von
Hildegard von Bingen gerne als stopfendes und zusammenziehendes Mittel bei Durchfallerkrankungen empfohlen. In der Praxis der Autorin sind getrocknete Heidelbeeren gerade bei Kindern, die an Diarrhö leiden, das Mittel erster Wahl. Auch bei BrechDurchfall-Erkrankungen werden sie gerne eingesetzt, da sie zusätzlich zur stopfenden auch eine brechreizlindernde Wirkung haben. Getrocknete Heidelbeeren sind im Fachhandel erhältlich oder können selber gesammelt und getrocknet werden $[3,4]$.

Inhaltsstoffe: Gerbstoffe, Proanthocyanidine, Anthocyane, Flavonoide, Invertzucker, Pektine, Mineralstoffe, Fruchtsäuren und Vitamine [3,5].

Wirkungen:Stopfend, entzündungshemmend, zusammenziehend, brechreizlindernd, keimhemmend $[3,5]$.

Darreichungsform: Getrocknete Beeren kauen $[3,5]$.

\section{Karotten (Daucus carota ssp. sativus)}

Eine lange gekochte Karottensuppe ist ein ideales Mittel zur Behandlung von Diarrhö. Diese Erkenntnis wird Dr. E. Moro (1874-1951), Direktor der Heidelberger Kinderklinik, zugeschrieben. Vermutlich ist die Karottensuppe aber in der Volksheilkunde schon viel länger bekannt. Dr. Moro und viele Kinderärzte, die seinem Beispiel folgten, hatten gute Erfolge bei der Behandlung von Kindern, die an Durchfall litten. Die Sterbe- und Komplikationsrate sank deutlich. Mit der

\section{KARGER}

๑) 2017 S. Karger GmbH, Freiburg 


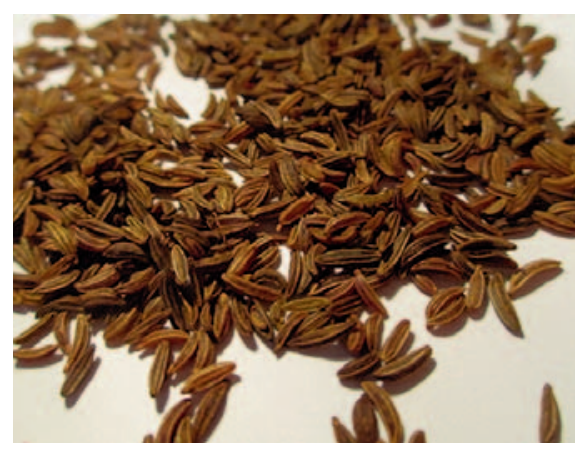

Abb. 1. Getrocknete Kümmelfrüchte (Carvi fructus).

Möglichkeit, Antibiotika zu verordnen, geriet die «Morosuppe», wie sie auch genannt wurde, aber nach und nach in Vergessenheit.

Die beeindruckende Wirkung der Karottensuppe wird heute mit dem Oligogalacturonid, einem Oligosaccharid, das in den Karotten enthalten ist, erklärt. Oligogalacturonide hemmen schon in kleiner Konzentration die Anheftung schädlicher Keime an der Darmschleimhaut. Beim Kochen werden die Oligogalacturonide, die den Rezeptoren der Darmmukosa stark ähneln, frei. Die Krankheitserreger binden dadurch an die Zuckermoleküle statt an die Darmmukosa an und können damit einfach ausgeschieden werden. Die Karottensuppe wirkt damit kausal und ersetzt mit dem zugegebenen Salz zugleich auch die verlorenen Elektrolyte [3].

Darreichungsform und Zubereitung: 500 g Karotten aus Bio-Anbau im Mixer pürieren, 1 Liter Wasser zugeben, 1-2 h köcheln lassen (bis sich die Flüssigkeitsmenge auf ca. $200 \mathrm{ml}$ reduziert hat). Danach durch ein Sieb streichen und in 11 Gemüsebrühe einrühren. Die lange Kochzeit ist zwingend, da die Oligogalacturonide erst nach längerem Kochen verfügbar werden. Die Suppe wird über den Tag verteilt in kleinen Mengen verabreicht und ist bereits für Säuglinge ab $6 \mathrm{Mo-}$ naten geeignet [3].

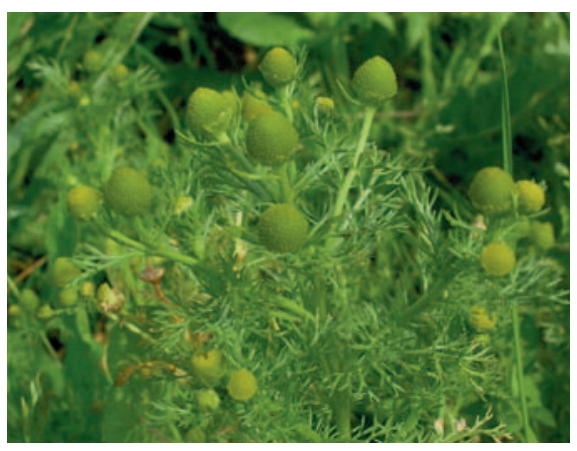

Abb. 2. Kamille im Frühstadium ihres Wachstums.

\section{Kü-Ka-Lei-Wasser}

Kü-Ka-Lei-Wasser (Kümmel-Kartoffel-Leinsamen-Wasser) wirkt blähungswidrig, entsäuernd, entzündungshemmend sowie harntreibend, pflegt die Schleimhaut und gibt ein wohliges Bauchgefühl.

Darreichungsform und Zubereitung: Eine Kartoffel in kleine Stücke schneiden, zusammen mit 1 TL Kümmelkörner (ungemahlen) (Abb. 1), 2 TL Leinsamen (ungeschrotet) und 1 Liter Wasser in eine Pfanne geben und 20 Minuten leicht köcheln lassen. Danach abseihen und über den Tag verteilt trinken (1. Gabe morgens 15-30 min vor dem Frühstück). Es wird empfohlen, das Kü-Ka-Lei-Wasser kurmässig 2-4 Wochen einzunehmen. Danach wird die Einnahme entweder beendet oder auf die eine Tasse KüKa-Lei-Wasser morgens auf nüchternen Magen reduziert [3, 5].

\section{Kamille (Matricaria recutita, Matricariae flos)}

Der gute, alte Kamillentee ist ein altbekanntes und weit verbreitetes Heilmittel für Magen-Darm-Verstimmungen und wird darum wohl auch immer wieder unterschätzt und abgewertet. Als universales Schleimhautmittel kann Kamille (Abb. 2) bei allen krampfartigen oder entzündlichen Magen-Darm-Beschwerden eingesetzt werden. Kamille wird auch erfolgreich bei Magenulkus eingesetzt

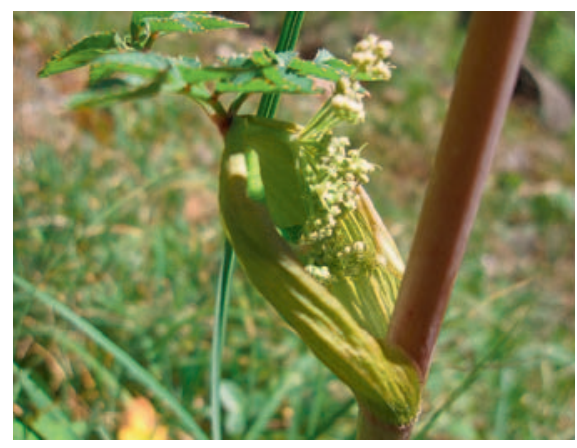

Abb. 3. Engelwurz ist seit jeher eine Kraft- und Schutzpflanze.

- sowohl in der Behandlung als auch in der Prävention [2-6].

Das Wesen der Kamille ist Sanftmut, Mütterlichkeit, Geborgenheit und milde Wärme. Darum wird die Kamille gerne auch verwendet, wenn ein Mensch unter übersteigerten inneren und äusseren Sinneswahrnehmungen leidet und mütterliche Geborgenheit, auch im übertragenen Sinne, sucht [7].

Inhaltsstoffe: Ätherisches Öl (mit Chamazulen und $\mathrm{x}$-Bisabolol), Schleimstoffe, Flavonoide und Cumarine $[2,3,5]$.

Wirkungen: Karminativ, entzündungshemmend, spasmolytisch, ulkusprotektiv, beruhigend, reizmildernd, antibakteriell, pilzhemmend, immunstimulierend $[2,3,5]$.

Darreichungsform: Tee, Tinktur [2, $3,5]$.

Eine besondere Anwendung der Kamille ist die sogenannte Rollkur. Dazu werden 2 Tassen sehr starker Kamillentee am Morgen nüchtern, noch im Bett liegend getrunken. Dann muss ca. 5-10 min auf dem Rücken, danach 5-10 Minuten auf der linken Seite, dann auf dem Bauch liegen geblieben und dann auf die rechte Seite «gerollt» werden. Auf diese Weise wird die Magenschleimhaut auf allen Seiten mit dem Kamillentee benetzt. Danach noch etwa 30 min nachruhen, am besten mit einem warmen Leibwickel. Tagsüber werden zusätzlich täglich 2-3 Tassen Kamillentee (ohne Rollprozedere) empfohlen. Die Rollkur wird etwa 10 Tage lang durchgeführt [3]. 


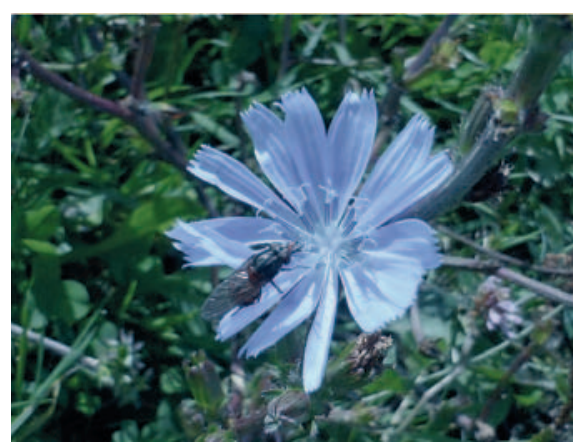

Abb. 4. Die Wegwarte ist eine der wenigen blauen Wildblumen.

\section{Echte Engelwurz (Angelica archangelica, Angelicae radix)}

Der Legende nach erhielt die Engelwurz (Abb. 3) ihren Namen, als ein Erzengel einem frommen Mann während der Pestepidemie die Pflanze als Heilmittel zeigte. Ursprünglich in Nordeuropa beheimatet, ist die Engelwurz aus der Familie der Doldenblütler (Apiaceae) eine hochangesehene Heilpflanze, wie viele volkstümliche Namen wie Angstwurz, Brustwurz, Heiligenbitter, Theriakwurz, Zauberwurz, Heiliger-Geist-Wurz, Zahnwurz, Glückwurz, Liebeswurz und Erzengelkraft zeigen. Heute wird Angelika vor allem bei Verdauungsstörungen, Husten und Schwächezuständen eingesetzt und gilt als einheimisches Adaptogenum. In Mitteleuropa ist auf feuchten Wiesen, an Waldrändern und an Uferböschungen oft ihre kleine Schwester, die Waldengelwurz (Angelica sylvestris), anzutreffen, die identisch angewendet wird.

Engelwurz ist seit jeher eine Kraftund Schutzwurzel. Als schutzmagischer Zauber wurde die Wurzel in früheren Zeiten als Amulett um den Hals getragen, um böse Geister und Dämonen fernzuhalten.

Das Wesen der Angelika ist es, den ureigenen Kraftquell zu zünden, damit der Mensch festen Boden unter den Füssen spüren kann und ein wacher und klarer Geist den Kopf mit den Wurzeln verbindet. Sie gibt dem Menschen den Mut, den eigenen Weg zu gehen - mit dem Kopf im Himmel

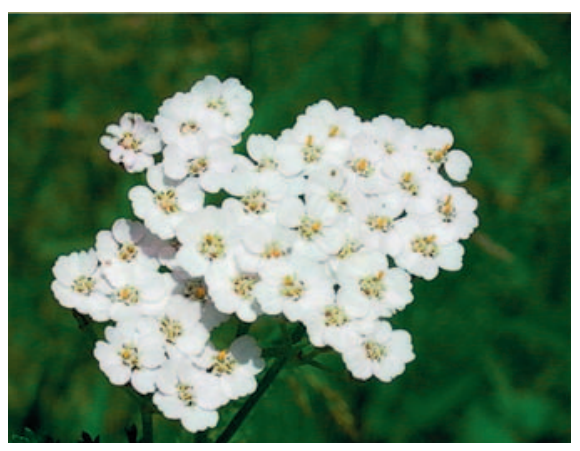

Abb. 5. Die Schafgarbe wird auch «Heil aller Welt» genannt.

und den Füssen gut geerdet auf dem Boden [2-6].

Im Magen-Darm-Trakt lindert sie Verdauungsschwäche, Dyspepsie, krampfartige Schmerzzustände und Appetitlosigkeit. Engelwurz bewährt sich im Speziellen bei Verdauungsbeschwerden, die mit Schwäche- und Erschöpfungszuständen einhergehen.

Inhaltsstoffe: Ätherische Öle, Bitterstoffe, Gerbstoffe, Pflanzensäuren, Cumarine, Furanocumarine [2, 3, 5].

Wirkungen: Spasmolytisch, karminativ, appetitanregend, magensaftsekretionsanregend, pankreasanregend, cholagog, antimikrobiell, menstruationsanregend, leicht stimmungsaufhellend [2, 3, 5].

Kontraindikationen: Schwangerschaft $[2,3,5]$.

Darreichungsform: Tee, Tinktur, oft als Bestandteil von traditionellen Hausrezepturen wie Kraftwein, Magenbitter usw. [2, 3, 5].

\section{Wegwarte (Cichorium intybus, Cichorii radix)}

Die Wegwarte (Abb. 4) leuchtet mit ihren hellblauen Blüten an Wegrändern, auf Schuttplätzen und auf Unkrautfluren. Es gibt nicht viele blaue Wildblumen und noch weniger blaue Korbblütler (Asteraceae). Ihre blauen Blüten richtet die Wegwarte immer der Sonne entgegen. Eine einzelne Blüte blüht nur einen Tag lang und nur bei Sonnenschein. In der alten $\mathrm{Na}$ turheilkunde ist die Pflanze als kräfti- gendes, verdauungsförderndes, blutreinigendes, harntreibendes, die Magensaftsekretion und den Gallenfluss förderndes Mittel bekannt. Die Heilkraft der Wegwarte war auch als Mittel gegen Melancholie hoch geschätzt. Klassische Anwendungsgebiete sind heute Verdauungsbeschwerden, Entgiftung über die Leber, Anregung des Gallenflusses, funktionelle Oberbauchbeschwerden, verdauungsbedingte Kopfschmerzen und Milzbeschwerden [2, 3, 5].

Die Wegwarte hat die Kraft, den Menschen mit beiden Beinen ins Jetzt dieses Augenblicks zu stellen, ihn aus der Schwärmerei zu wecken und ihn mit dem Moment zu verbinden. Nicht durch die Vergangenheit, nicht durch die Zukunft, sondern durch den jetzigen Moment ist der Mensch mit seinem inneren Wissen verbunden. Treten Magen-Darm-Beschwerden aufgrund von schwierigen Lebenssituationen auf, ist die Wegwarte Mittel erster Wahl, da sie den Menschen (wieder) sein inneres Ich spüren lässt.

Volkstümliche Namen der Wegwarte wie Sonnenbraut oder Sonnenwirbel zeigen die mit Leichtigkeit erfüllte Erhellung. Für die vorchristlichen Völker in Europa war die Wegwarte eine pflanzliche Verkörperung der weissen Göttin, die nach ihrem Geliebten Ausschau hält. Ihr Geliebter ist nach ihrer Mythologie der Sonnengott und der Hirschkönig mit seinen Attributen Gold und Hirschhorn [5].

Inhaltsstoffe: Bitterstoffe, Cichoriensäure, Inulin, Pentosane [2, 3, 5].

Wirkungen: Appetit- und verdauungsanregend, gallenflussanregend, cholesterinsenkend $[2,3,5]$.

Darreichungsform: Tee, Tinktur, oft als Bestandteil von traditionellen Hausrezepturen wie Kraftwein, Magenbitter usw. [2, 3, 5].

\section{Schafgarbe (Achillea millefolium, Millefolii herba/flos)}

Die Volksheilkunde und alle alten Kräuterbücher loben die Schafgarbe in 
hohen Tönen - sie wird auch «Heil aller Welt» genannt (Abb. 5). Neben ihrer Verwendung in der Wundpflege und der Frauenheilkunde ist sie eine wichtige Heilpflanze für den MagenDarm-Trakt, wovon auch ihr Name «Bauchwehkraut» herrührt. Da sie unter den Bitterstoffpflanzen zu den milderen gehört und ihre Wirkprinzip oft als «Harmonisierung» beschrieben wird, kann sie bei (fast) allen Verdauungsbeschwerden gut eingesetzt werden. In Mittel- und Nordeuropa ist sie auf trockenen Wiesen sowie an Ackerund Wegrändern zu finden. Sie ist eine wichtige Pionierpflanze [2-6].

Roger Kalbermatten schreibt über die Schafgarbe: «Das Wesen der Schaf- garbe symbolisiert das Vermögen zur Unterscheidung. Bei der richtigen Unterscheidung, die jeder guten Entscheidung vorangeht, müssen wir immer die gegensätzlichen Pole einer Sache erkennen, auseinanderhalten und im Zusammenhang beurteilen. Gelingt dies nicht, so fallen wir abwechselnd von extremer Zustimmung in extreme Ablehnung und die richtige Wahl oder der goldene Mittelweg bleibt verschlossen. Die Kraft der Schafgarbe liegt darin, uns aus den polaren Eigenschaften des Lebens die richtige Erkenntnis ziehen zu lassen» [6]. Diese Thematik ist der Schafgarbe nicht nur im energetischen Sinn zu eigen, sondern auch ganz konkret auf die Verdauung bezogen, wenn es darum geht, Nahrung in rechtem Mass zu verstoffwechseln und zu entscheiden, welche Nahrungsbestandteile resorbiert und welche ausgeschieden werden sollen.

Inhaltsstoffe: Ätherische Öle, Bitterstoffe, Gerbstoffe, Flavonoide [2, 3, 5].

Wirkungen: Appetit- und gallenflussfördernd, mild entgiftend, spasmolytisch, tonisierend, karminativ, antibakteriell, entzündungshemmend und das Leberparenchym regenerierend $[2,3,5]$.

Darreichungsform: Tee, Tinktur, ausserdem häufiger Bestandteil vieler Magen-Darm-Therapeutika [2, 3, 5].

\section{Literatur}

1 Raimann C, Ganz C, Garvelmann F, BertschiStahl HD, Fehr-Streule R: Grundlagen der traditionellen europäischen Naturheilkunde. Schiedlberg, Bacopa, 2012.

2 Garvelmann F, Raimann C: Humoralmedizinsche Praxis. Schiedlberg, Bacopa, 2016.

3 Bühring U: Praxis-Lehrbuch Heilpflanzenkunde. Stuttgart, Haug, 2014.

4 Künzle J: Das grosse Kräuter-Heilbuch. Olten, Otto Walter, 1945.

5 Bühring U: Alles über Heilpflanzen. Stuttgart, Ulmer, 2011.
6 Treben M: Heilkräuter aus dem Garten Gottes. München, Heyne, 1986.

7 Kalbermatten R: Wesen und Signatur der Heilpflanzen. Aarau, AT, 2002. 